\title{
Empirical Formulas of Shear Modulus and Damping Ratio for Geopolymer-Stabilized Coarse-Grained Soils
}

\author{
Shengnian Wang ${ }^{1}$, Xinqun Gao ${ }^{1}$, Wei $\mathrm{Ma}^{2}$, Guoyu $\mathrm{Li}^{2 *}$, Chong Shi ${ }^{3}$ and Peng Zhang ${ }^{1}$ \\ ${ }^{1}$ College of Transportation Science and Engineering, Nanjing Tech University, Nanjing, China, ${ }^{2}$ State Key Laboratory of Frozen \\ Soil Engineering, Northwest Institute of Eco-Environment and Resources, Chinese Academy of Sciences, Lanzhou, China, \\ ${ }^{3}$ Institute of Geotechnical Engineering, Hohai University, Nanjing, China
}

OPEN ACCESS

Edited by:

Wanqing Shen,

Université de Lille, France

Reviewed by:

Lanlan Yang,

Jiangnan University, China

Jun Yu,

Nantong University, China

*Correspondence:

Guoyu Li

guoyuli@/zb.ac.cn

Specialty section:

This article was submitted to Interdisciplinary Physics, a section of the journal

Frontiers in Physics

Received: 06 August 2021 Accepted: 01 September 2021 Published: 25 November 2021

Citation:

Wang S, Gao X, Ma W, Li G, Shi C and Zhang P (2021) Empirical Formulas of Shear Modulus and Damping Ratio for Geopolymer-Stabilized Coarse-

Grained Soils.

Front. Phys. 9:754377.

doi: 10.3389/fphy.2021.754377
The contribution of gravel fraction on the maximum shear modulus $\left(G_{\text {max }}\right)$, dynamic shear modulus ratio $\left(G / G_{\text {max }}\right)$, and damping ratio $(\lambda)$ of cementitious coarse-grained soils has not been fully understood yet. Large-scale triaxial cyclic tests for geopolymer-stabilized coarse-grained soils (GSCGSs) were conducted with different volumetric block proportions (VBPs) under various confining pressures (CPs) for investigating their dynamic behaviors and energy dissipation mechanisms. Results indicate that the $G_{\max }$ of GSCGS increases linearly with VBPs but nonlinearly with CP. High VBPs will probably result in a gentle decrease in $G / G_{\max }$ and a rapid increase in normalized $\lambda\left(\lambda_{\text {nor }}\right)$, while the opposite is the case for a high $\mathrm{CP}$. With the shear strain amplitude being normalized, the $\mathrm{G} /$ $G_{\text {max }}$ and $\lambda_{\text {nor }}$ are distributed in a narrow band with low dispersion and thus can be welldescribed by empirical functions of the normalized shear strain amplitude.

Keywords: coarse-grained soils, geopolymer, shear modulus, damping ratio, empirical formulas

\section{INTRODUCTION}

Cementitious coarse-grained soils (CCGSs) are widely used as filling materials in infrastructure projects such as high-speed railway subgrades, earth dams, and highways [1,2]. However, the design and construction of engineering structures on CCGS are always challenging for engineers due to parameter determination difficulties. Dynamic soil properties including the maximum shear modulus $\left(G_{\max }\right)$, dynamic shear modulus ratio $\left(G / G_{\max }\right)$, and damping ratio $(\lambda)$ from small to large shear strain amplitude $(\gamma)$ are crucial indices for the seismic design and stability evaluation of geotechnical structures subjected to periodic random loads. Previous studies showed that CCGS was inhomogeneous and heterogeneous geotechnical materials [1,2]. Their cyclic shear behaviors were affected by gravel fraction, cementation, interparticle contact stiffness, void ratio, curing period, and deformation within individual particles [3-6]. Of these factors, the gravel fraction and cementation played a particularly significant role in the shear behavior of CCGS. However, no consensus exists on their effects up to now. Geopolymer binders (GBs) are alkali-activated aluminosilicate gel materials with enormous advantages in high strength, fast hardness, weak shrinkage, etc. Their primary raw materials are solid wastes, such as fly ash, glass waste, red mud, metakaolin (MK), and combinations of two or more of these materials [7]. The coarse-grained soil stabilized with GBs (GSCGS) thus can also be a better choice for engineering practices, regardless of safety performance in seismic resistance and durability or feasibilities in resource acquisition and cost control. This study conducted large-scale undrained triaxial cyclic tests on GSCGS with different volumetric block 

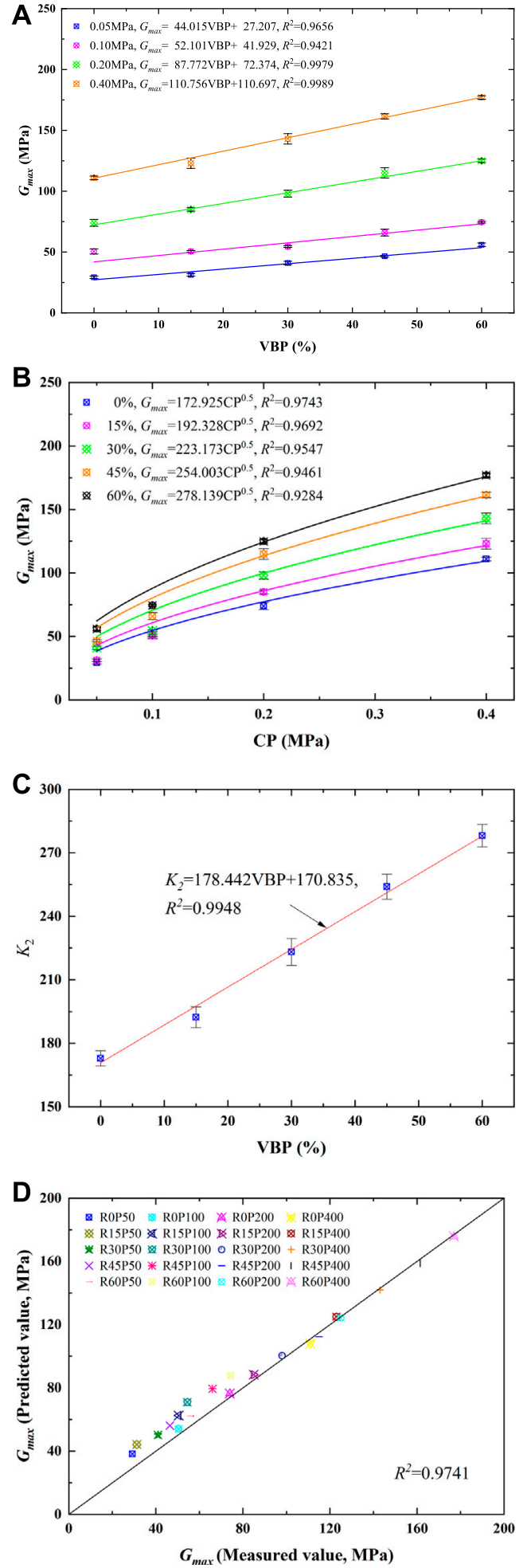

FIGURE 1 | Relationships of the $G_{\max }$ of GSCGS with the VBP and CP.

proportions (VBPs) under various confining pressures (CPs). The evolution of $G_{\max }, G / G_{\max }$, and $\lambda$ was investigated, and their relationships with $\gamma$ were discussed.

\section{EXPERIMENTS}

The dynamic behaviors of GSCGS in this study were investigated via a large-scale triaxial cyclic shear instrument (HCA300) developed by the American company GCTS. Each GSCGS cylindrical specimen was $100 \mathrm{~mm}$ in diameter and $200 \mathrm{~mm}$ in height. For the convenience of sample preparation, coarsegrained soils were considered a mixture of the soil matrix and rock blocks. The soil matrix was fine-grained residual soil, with a maximum grain size of $2 \mathrm{~mm}$. The natural dry density was $1.64 \mathrm{~g} /$ $\mathrm{cm}^{3}$. The maximum dry density and optimum water content were $1.72 \mathrm{~g} / \mathrm{cm}^{3}$ and $18.3 \%$, respectively. The rock blocks mainly comprised crushed stones with a dry density of $2.42 \mathrm{~g} / \mathrm{cm}^{3}$. The maximum rock block size was limited to be 0.2 times the diameter of the specimen to avoid the grain size effect, namely, the rock block size used in sample preparation was $2-20 \mathrm{~mm}$.

Considering that the VBP greater than $60 \%$ may result in considerable hollow phenomena among rock blocks and significant difficulties in packing GSCGS samples in the mold, only five VBPs $(0 / 15 / 30 / 45 / 60, \%)$ combined with four CPs $(0.05 /$ $0.10 / 0.20 / 0.40, \mathrm{MPa})$ were considered in this study. The previous study showed that GBs could synthesize from $\mathrm{MK}, \mathrm{CaO}$, and $\mathrm{NaHCO}_{3}$ with a mass ratio of $4: 1: 1$, and their optimal mixing ratio in fine-grained soil was $15 \mathrm{wt} \%$ [7]. Therefore, the dosage of GBs in the coarse-grained soil samples was determined by the relative content of the soil matrix because of the cementation of GB functions primarily in the fine-grained soil. In other words, once the VBP is selected, the dosage of fine-grained soil in a GSCGS specimen is known, and the dosage of GBs can be determined. The water consumption for sample preparation was the sum of the amount of water required for the fine-grained soil to reach its maximum dry density and an extra water compensation of $5 \%$ for rock blocks' water absorption. All the specimens were cured in a humid environment at room temperature for 7 days and saturated by a vacuum extractor on GCTS until the B-value reached 0.95 at least before loading. The axial strain amplitude was increased from $1 \times 10^{-5}$ to $1 \times 10^{-2}$ in a level-by-level manner. The number of cyclic loadings for each strain amplitude was 5 . The loading frequency was $0.5 \mathrm{~Hz}$.

\section{RESULTS AND DISCUSSION}

Dynamic soil properties, including $G$ and $\lambda$, were achieved by following the calculation methods for symmetrical and asymmetric hysteresis loops suggested by Kumar et al. [8]. Figure 1A presents the relationship between the $G_{\max }$ of GSCGS and the VBP. The $G_{\text {max }}$ always increases linearly with the VBP, despite GSCGS being subjected to tensile or compressive stress. The increasing gradient of fitting curves suggests that there is a positive correlation between the $G_{\text {max }}$ and CP. Hence, the relationship of the $G_{\text {max }}$ and VBP can be described as follows:

$$
G_{\text {max }}=k_{p} V B P+G_{\text {matrix }},
$$

where $k_{p}$ is the gradient of fitting curves and $G_{\text {matrix }}$ is the intercept denoting the fundamental stiffness of the soil matrix under a specified CP. The fitting results based on Eq. 1 illustrate that the $k_{p}$ 

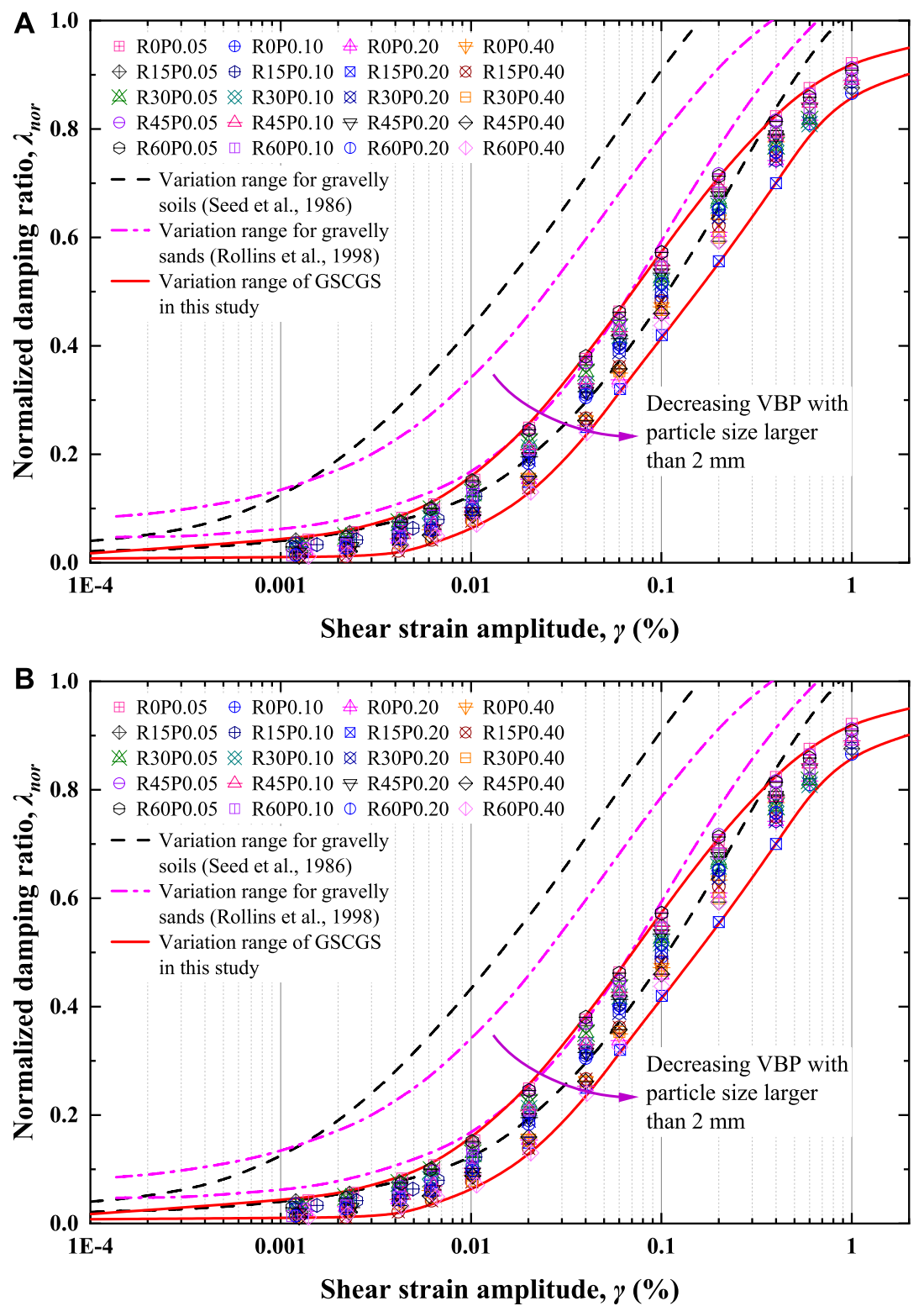

FIGURE 2 | G/G $G_{\max }$ and $\lambda$ envelope curves of GSCGS with different VBPs under various CPs.

increases with the $\mathrm{CP}$, namely, high $\mathrm{CP}$ will result in larger values in $G_{\text {max }}$. Figure $1 B$ presents the relationship between the $G_{\max }$ of GSCGS and the CP. The $G_{\max }$ increases nonlinearly with the CP at the same VBP. Seed et al. [9] proposed a simplified relationship between the $G_{\max }$ and CP for gravelly soil as follows:

$$
G_{\text {max }}=K_{2}(C P)^{0.5} \text {, }
$$

where $K_{2}$ is a regression coefficient. Rollins et al. [10] reported that $K_{2}$ was a function of relative density for soils. Since the GSCGS is regarded as the soil matrix and rock blocks, the density of GSCGS can be summarized as a function of the VBP. Therefore, $K_{2}$ is related to the VBP of GSCGS. Figure 1C illustrates an excellent linear correlation between $K_{2}$ and VBP. Thus, a new empirical formula for the $G_{\max }$ of GSCGS is defined as follows:

$$
G_{\text {max }}=\left(k_{0} V B P+C\right)(C P)^{0.5},
$$

where $k_{0}$ and $C$ are regression coefficients. Figure 1D presents the measured and predicted $G_{\max }$ of GSCGS. Both are close to the bisecting line with a high correlation coefficient $\left(R^{2}\right)$ of 0.9741 , which indicates that the proposed empirical formula can predict the $G_{\max }$ of GSCGS well.

Figure 2A presents the $G / G_{\max }$ envelope curves of GSCGS with different VBPs under various CPs. The $G / G_{\text {max }}$ is distributed within a band on the whole. The shape of the curves is very close as $\gamma$ is less than the order of $10^{-4} \%$. When $\gamma$ lies between $10^{-4} \%$ and $0.01 \%$, the $G / G_{\max }$ is scattered. When $\gamma$ lies between 0.01 and $1.0 \%$, the $G / G_{\text {max }}$ decreases significantly. The reduction rate of $G /$ $G_{\text {max }}$ slows down once $\gamma$ is higher than $1.0 \%$. As a whole, the $G /$ 


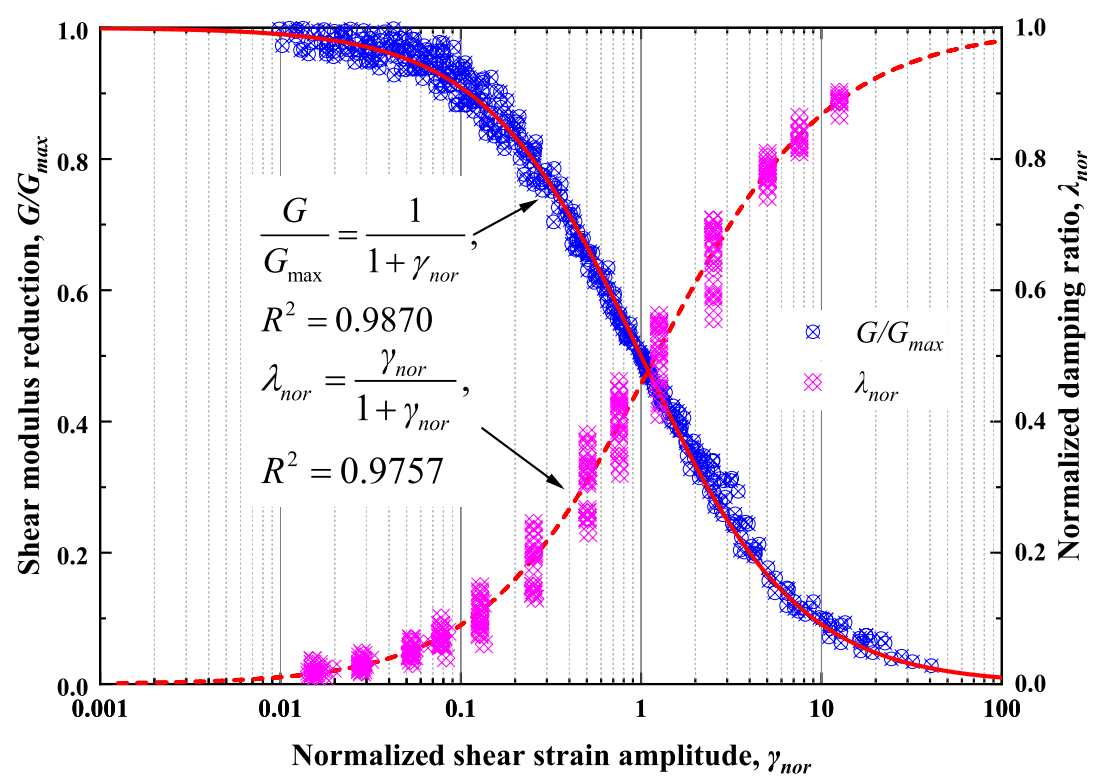

FIGURE 3 | Relationships of the G/G $G_{\max }$ and $\lambda_{\text {nor }}$ of GSCGS with $\gamma_{\text {nor }}$.

$G_{\text {max }}$ of GSCGS is more likely to be characterized following a hyperbolic $G / G_{\max }$ function proposed by Hardin and Drnevich [11], which is given in the following equation:

$$
G / G_{\max }=1 /\left(1+\gamma / \gamma_{r}\right)^{n}
$$

where $\gamma_{r}$ is the reference shear strain and $n$ is the curvature coefficient. It can be observed that the envelope region of $G$ / $G_{\text {max }}$ overlaps with the bounds proposed by Rollins et al. [10] when the VBP of GSCGS is higher than $45 \%$. However, when the VBP is less than $45 \%$, they have not overlapped anymore, especially when $\gamma$ ranges between 0.01 and $1.0 \%$. Seed et al. [9] pointed out that the $G / G_{\text {max }}$ of sands always decreased faster than gravelly soils as $\gamma$ increased, namely, high VBP would result in a gentle decrease in $G$ / $G_{\max }$ of gravelly soils. This discovery explains why the $G / G_{\max }$ envelope curves of GSCGS are relatively higher than those of gravelly soils used in studies by Seed et al. [9] and Rollins et al. [10].

Figure 2B shows the normalized $\lambda\left(\lambda_{\text {nor }}\right)$ envelope curves of GSCGS with different VBPs under various CPs, wherein the empirical model proposed by Chen et al. [12] is applied.

$$
\lambda_{\text {nor }}=\lambda_{0}\left(1-G / G_{\max }\right)^{n} /\left(\lambda_{\max }-\lambda_{\min }\right),
$$

where $\lambda_{\min }$ and $\lambda_{\max }$ are the minimum and maximum $\lambda$, respectively, and $\lambda_{0}$ and $n$ are regression parameters related to soil properties. It can be observed that $\lambda_{\text {nor }}$ is distributed in a narrower band overall. The shape of the curves becomes unanimous when $\gamma$ is less than the order of $10^{-3} \%$. This result implies that the VBP and CP might have a minimal impact on $\lambda_{\text {nor }}$. The reason why the $\lambda_{\text {nor }}$ envelope curves of GSCGS are lower than those of gravelly soils examined by Seed et al. [9] and Rollins et al. [10] maybe that a high VBP is more likely to result in significant difficulties in compaction of coarse-grained soils, while cementation improves the integrity of CGS significantly, and thereby results in relatively low $\lambda_{\text {nor }}$ when subjected to cyclic loadings.
Figure 3 presents the relationship of the $G / G_{\text {max }}$ and $\lambda_{\text {nor }}$ of GSCGS vs. normalized $\gamma\left(\gamma_{\text {nor }}=\gamma / \gamma_{r}\right)$. It can be observed that both $G / G_{\text {max }}$ and $\lambda_{\text {nor }}$ are distributed within a narrow band, namely, both of them are insensitive to the VBP and CP via $\gamma_{\text {nor }}$. Martin and Seed [13] had summarized a nonlinear elastic model for gravel soils with $\gamma_{\text {nor }}$, which is

$$
G / G_{\max }=1-\left[{\gamma_{\text {nor }}}^{2 \beta} /\left(1+\gamma_{\text {nor }}{ }^{2 \beta}\right)\right]^{\alpha},
$$

where $\alpha$ and $\beta$ are regression parameters. The fitting results of $G /$ $G_{\text {max }}$ show that this nonlinear model is also available to GSCGS with an excellent correlation coefficient of 0.9870 and can be simplified as follows:

$$
G / G_{\max }=1 /\left(1+\gamma_{\text {nor }}\right) .
$$

Substituting Eqs 4, 6 into Eq. 5 yields

$$
\lambda_{\text {nor }}=\left[\gamma_{\text {nor }}^{2 \beta} /\left(1+\gamma_{\text {nor }}^{2 \beta}\right)\right]^{\alpha n} .
$$

The fitting results of $\lambda_{\text {nor }}$ show a perfect correlation of 0.9757 with $\gamma_{\text {nor }}$, and can be rewritten as follows:

$$
\lambda_{\text {nor }}=\gamma_{\text {nor }} /\left(1+\gamma_{\text {nor }}\right) \text {. }
$$

This empirical formula thus can characterize $\lambda$ of GSCGS under cyclic loadings.

\section{CONCLUSION}

The dynamic properties of GSCGS were investigated via largescale triaxial cyclic tests in this study. Outcomes illustrate that the $G_{\text {max }}$ of GSCGS increases linearly with the VBP but nonlinearly with CP. Thus, new empirical formulas of $G_{\text {max }}$ referring to the VBP and CP are proposed. A high VBP may result in a gentle 
decrease in $G / G_{\text {max }}$ and a rapid increase in $\lambda_{\text {nor }}$, while the opposite is the case for a high CP. $G / G_{\text {max }}$ and $\lambda_{\text {nor }}$ are insensitive to VBP and $\mathrm{CP}$ via $\gamma_{\text {nor }}$ so that they can be described by empirical formulas of $\gamma_{\text {nor }}$. The proposed empirical formulas can provide a reference to understand the dynamic behaviors of GSCGS and other similar cementitious geomaterials.

\section{DATA AVAILABILITY STATEMENT}

The original contributions presented in the study are included in the article/Supplementary Material; further inquiries can be directed to the corresponding author.

\section{REFERENCES}

1. Wang T-1., Wang H-h., Song H-f., Yue Z-r., and Guo Z-h. Effects of Cement Content and Grain-Size Composition on Engineering Properties of HighSpeed-Railway Macadam Subgrade. Cold Regions Sci Tech (2018) 145:21-31. doi:10.1016/j.coldregions.2017.09.009

2. Fu Z, Chen S, and Han H. Large-scale Triaxial Experiments on the Static and Dynamic Behavior of an Artificially Cemented Gravel Material. Eur J Environ Civil Eng (2020) 2020:1-21. doi:10.1080/ 19648189.2020.1792350

3. Pestana JM, and Salvati LA. Small-Strain Behavior of Granular Soils. I: Model for Cemented and Uncemented Sands and Gravels. J Geotech Geoenviron Eng (2006) 132(8):1071-81. doi:10.1061/(asce)1090-0241(2006)132:8(1071)

4. Wang H-L, Cui Y-J, Lamas-Lopez F, Calon N, Saussine G, Dupla J-C, et al. Investigation on the Mechanical Behavior of Track-Bed Materials at Various Contents of Coarse Grains. Construction Building Mater (2018) 164:228-37. doi:10.1016/j.conbuildmat.2017.12.209

5. Liu X, Zhang X, Wang H, and Jiang B. Laboratory Testing and Analysis of Dynamic and Static Resilient Modulus of Subgrade Soil under Various Influencing Factors. Construction Building Mater (2019) 195:178-86. doi:10.1016/j.conbuildmat.2018.11.061

6. Xu D-s., Liu H-b., Rui R, and Gao Y. Cyclic and Postcyclic Simple Shear Behavior of Binary Sand-Gravel Mixtures with Various Gravel Contents. Soil Dyn Earthquake Eng (2019) 123:230-41. doi:10.1016/ j.soildyn.2019.04.030

7. Wang S, Xue Q, Zhu Y, Li G, Wu Z, and Zhao K. Experimental Study on Material Ratio and Strength Performance of Geopolymer-Improved Soil. Construction Building Mater (2021) 267:120469. doi:10.1016/ j.conbuildmat.2020.120469

8. Kumar SS, Krishna AM, and Dey A. Evaluation of Dynamic Properties of sandy Soil at High Cyclic Strains. Soil Dyn Earthquake Eng (2017) 99:157-67. doi:10.1016/j.soildyn.2017.05.016

\section{AUTHOR CONTRIBUTIONS}

Funding acquisition and formal writing of the work, SW; investigation and data analysis of the work, XG; review and editing of the work, WM, GL, CS, and PZ. All authors have read and agreed to the published version of the manuscript.

\section{FUNDING}

This study was financially supported by the National Natural Science Foundation of China (41902282) and State Key Laboratory of Frozen Soil Engineering (SKLFSE201809).

9. Seed HB, Wong RT, Idriss IM, and Tokimatsu K. Moduli and Damping Factors for Dynamic Analyses of Cohesionless Soils. J Geotechnical Eng (1986) 112(11):1016-32. doi:10.1061/(asce)0733-9410(1986)112:11(1016)

10. Rollins KM, Evans MD, Diehl NB, and Iii WDD. Shear Modulus and Damping Relationships for Gravels. J Geotechnical Geoenvironmental Eng (1998) 124(5): 396-405. doi:10.1061/(asce)1090-0241(1998)124:5(396)

11. Hardin BO, and Drnevich VP. Shear Modulus and Damping in Soils: Design Equations and Curves. J Soil Mech Foundations Div (1972) 98(sm7):667-92. doi:10.1061/jsfeaq.0001760

12. Chen G, Zhou Z, Sun T, Wu Q, Xu L, Khoshnevisan S, et al. Shear Modulus and Damping Ratio of Sand-Gravel Mixtures over a Wide Strain Range. J Earthquake Eng (2019) 23(8):1407-40. doi:10.1080/13632469.2017.1387200

13. Martin PP, and Seed HB. One-dimensional Dynamic Ground Response Analyses. J Geotech Engrg Div (1982) 108(7):935-52. doi:10.1061/ ajgeb6.0001316

Conflict of Interest: The authors declare that the research was conducted in the absence of any commercial or financial relationships that could be construed as a potential conflict of interest.

Publisher's Note: All claims expressed in this article are solely those of the authors and do not necessarily represent those of their affiliated organizations, or those of the publisher, the editors, and the reviewers. Any product that may be evaluated in this article, or claim that may be made by its manufacturer, is not guaranteed or endorsed by the publisher.

Copyright $\odot 2021$ Wang, Gao, Ma, Li, Shi and Zhang. This is an open-access article distributed under the terms of the Creative Commons Attribution License (CC BY). The use, distribution or reproduction in other forums is permitted, provided the original author(s) and the copyright owner(s) are credited and that the original publication in this journal is cited, in accordance with accepted academic practice. No use, distribution or reproduction is permitted which does not comply with these terms. 